\title{
A DYNAMICAL INVESTIGATION OF NINE WIDE VISUAL DOUBLE STARS IN THE NEIGHBOURHOOD OF THE SUN
}

\author{
A.A.KISSELEV AND L.G.ROMANENKO \\ Pulkovo Observatory, \\ 196140 St Petersburg, Russia, \\ e-mail: ddp@gaoran.spb.su
}

The dynamical states of nine wide visual double stars (ADS 7251, 10329, 10386, 10759 [psi Dra], 11061 [40\&41 Dra], 11632, 12169, 12815 [16 Cyg]) are considered. The 20-30 - year series of photographic observations obtained with the Pulkovo 26-inch refractor (Kisselev et al., 1988) supported by the data of relative radial velocities of the components and parallaxes are used. On this base the vectors of relative spatial positions and velocities of the components are determined or estimated with confidence. Families of orbits, satisfying observational data are determined by assuming stability of motions in the systems. The orbits of three nearby binaries ADS 7251, 11632 and 61 Cyg were calculated earlier by classical methods and belong to the family mentioned above. Thus the authenticity of our analysis is proved (Kisselev, Kiyaeva, 1980). It is shown that elliptical motion in the couples ADS 10759 and 11061 could be explained only if an additional (hidden) mass exists. This mass consists of one solar mass for the first couple and two solar masses for the second one. The dynamical orbital elements of the binaries belonging to each family may differ greatly, but the geometrical elements are fairly stable. The orientation of the orbit planes of binaries with respect to the Galaxy plane is determined. In one half of the cases the orbital planes are approximately orthogonal to that of our Galaxy.

\section{References}

Kisselev, A.A., et al. (1988) The catalogue of relative positions and motions for 200 visual double stars observed with 26-inch refractor at Pulkovo in 1960-1986. Nauka, Leningrad.

Kisselev, A.A., Kiyaeva, O.V., (1980), Applications of the Apparent Motion Parameters (AMP) Method for Determination of the Visual Double Star Orbits, Soviet. Astron., 57, pp. 1227-1241. 